\title{
Entropic Stochastic Resonance
}

\author{
P. S. Burada, ${ }^{1}$ G. Schmid, ${ }^{1}$ D. Reguera, ${ }^{2}$ M. H. Vainstein, ${ }^{2}$ J. M. Rubi, ${ }^{2}$ and P. Hänggi ${ }^{1}$ \\ ${ }^{1}$ Institut für Physik, Universität Augsburg, Universitätsstr. 1, D-86135 Augsburg, Germany \\ ${ }^{2}$ Departament de Física Fonamental, Facultat de Física, Universidad de Barcelona, Diagonal 647, E-08028 Barcelona, Spain
}

(Received 14 July 2008; published 25 September 2008)

\begin{abstract}
We present a novel scheme for the appearance of stochastic resonance when the dynamics of a Brownian particle takes place in a confined medium. The presence of uneven boundaries, giving rise to an entropic contribution to the potential, may upon application of a periodic driving force result in an increase of the spectral amplification at an optimum value of the ambient noise level. The entropic stochastic resonance, characteristic of small-scale systems, may constitute a useful mechanism for the manipulation and control of single molecules and nanodevices.
\end{abstract}

Stochastic resonance $(\mathrm{SR})$ describes the counterintuitive phenomenon where an appropriate dose of noise is not harmful for the detection or transduction of an incoming, generally weak signal, but rather of constructive use in the sense that a weak signal becomes amplified upon harvesting the ambient noise in metastable, nonlinear systems [1]. Since its first discovery in the early eighties SR has been observed in a great variety of systems pertaining to different disciplines such as physics, chemistry, engineering, biology and biomedical sciences [1-10]. The list of models and applications is still growing. In particular, SR has found widespread interests and applications within biological physics.

The research on SR has primarily been focused on systems with purely energetic potentials. However, in situations frequently found in soft condensed matter and biological systems, particles move in constrained regions such as small cavities, pores or channels whose presence and shape play an important role for the SR dynamics [10], sometimes even more important than the well-studied case of energetic barriers in such systems [11-14]. In this Letter, we demonstrate that irregularities in the form of confining, curved boundaries, being modeled via an entropic potential, can cause noise-assisted, resonantlike behaviors in the system under consideration. Confinement, an inherent property of small-scale systems, can thus constitute an important source of noise-induced resonant effects with interesting applications in the design and control of these systems.

The phenomenon of SR is rooted on a stochastic synchronization between noise-induced hopping events and the rhythm of the externally applied signal, that taken alone is not sufficient for the system to overcome a potential barrier. In the first place, noise enables system transitions and it is in fact responsible for the observed signal amplification and the emergence of a certain degree of order. In the earliest and basic manifestation of SR, the synchronization of the random switches of a Brownian particle with a periodic driving force was observed for a bistable potential.
Moreover, potentials of this type are not only found in systems with energy barriers, as they may also arise due to the influence of entropic constraints. Particles diffusing freely in a confined medium such as the one depicted in Fig. 1 may give rise to an activation regime when a constant force $\vec{G}$ in the transversal direction is imposed. We will show that the combination of forcing and the presence of entropic effects deriving from the confinement and the irregularity of the boundaries give rise to an effective bistable potential that exhibits the signatures of stochastic resonance.

The dynamics of a particle in a constrained geometry subjected to a sinusoidal oscillating force $F(t)$ along the axis of the structure and to a constant force $G$ in the transversal direction can be described by means of the Langevin equation, written in the overdamped limit as

$$
\gamma \frac{d \vec{r}}{d t}=-G \vec{e}_{y}-F(t) \vec{e}_{x}+\sqrt{\gamma k_{B} T} \vec{\xi}(t),
$$

where $\vec{r}$ denotes the position of the particle, $\gamma$ is the friction coefficient, $\vec{e}_{x}$ and $\vec{e}_{y}$ the unit vectors along the $x$ and $y$ directions, respectively, and $\vec{\xi}(t)$ is a Gaussian white noise with zero mean which obeys the fluctuation-dissipation

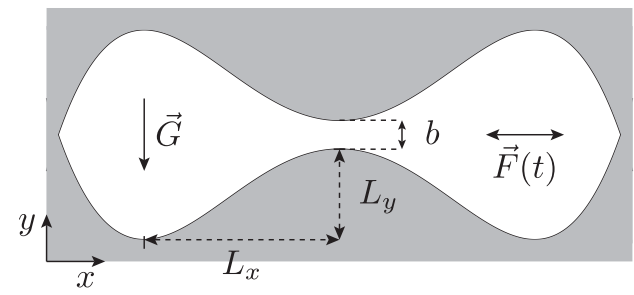

FIG. 1. Schematic illustration of the two-dimensional structure confining the motion of the Brownian particles. The symmetric structure is defined by a quartic double well function, cf. Eq. (2), involving the geometrical parameters $L_{x}, L_{y}$ and $b$. Brownian particles are driven by a sinusoidal force $\vec{F}(t)$ along the longitudinal direction and a constant force $\vec{G}$ in the transversal direction. 
relation $\left\langle\xi_{i}(t) \xi_{j}\left(t^{\prime}\right)\right\rangle=2 \delta_{i j} \delta\left(t-t^{\prime}\right)$ for $i, j=x, y$. The explicit form of the longitudinal force is given by $F(t)=$ $F_{0} \sin (\Omega t)$ where $F_{0}$ is the amplitude and $\Omega$ is the frequency of the sinusoidal driving.

In the presence of confining boundaries, this equation has to be solved by imposing reflecting boundary conditions at the walls of the structure. For the 2D structure depicted in Fig. 1, the walls are defined by

$$
w_{l}(x)=L_{y}\left(\frac{x}{L_{x}}\right)^{4}-2 L_{y}\left(\frac{x}{L_{x}}\right)^{2}-\frac{b}{2}=-w_{u}(x),
$$

where $w_{l}$ and $w_{u}$ correspond to the lower and the upper boundary functions, respectively, $L_{x}$ corresponds to the distance between bottleneck position and position of maximal width, $L_{y}$ refers to the narrowing of the boundary functions, and $b$ to the remaining width at the bottleneck, cf. Fig. 1. Consequently, $2 w(x)=w_{u}(x)-w_{l}(x)$ gives the local width of the structure. This choice of the geometry is intended to resemble the archetypical setup for SR in the context of a double well potential. In fact, in the limit of a sufficiently large transversal force, the particle is in practice restricted to explore the region very close to the lower boundary of the structure, recovering the effect of an energetic bistable potential. For the sake of a dimensionless description, we henceforth scale all lengths by the characteristic length $L_{x}$, i.e., $\tilde{x}=x / L_{x}, \tilde{y}=y / L_{x}$ which implies $\tilde{b}=b / L_{x}$ and $\tilde{w}_{l}=w_{l} / L_{x}=-\tilde{w}_{u}$, time by $\tau=$ $\gamma L_{x}^{2} / k_{B} T_{R}$, the corresponding characteristic diffusion time at an arbitrary, but irrelevant reference temperature $T_{R}$, i.e., $\tilde{t}=t / \tau$ and $\tilde{\Omega}=\Omega \tau$, and force by $F_{R}=\gamma L_{x} / \tau$, transversal force $\tilde{G}=G / F_{R}$ and a longitudinally acting, sinusoidal force $\tilde{F}(\tilde{t})=F(t) / F_{R}$. In the following we shall omit the tilde symbols for better legibility. In dimensionless form the Langevin equation (1) and the boundary functions (2) read:

$$
\begin{gathered}
\frac{d \vec{r}}{d t}=-G \vec{e}_{y}-F(t) \vec{e}_{x}+\sqrt{D} \vec{\xi}(t), \\
w_{l}(x)=-w_{u}(x)=-w(x)=\epsilon x^{4}-2 \epsilon x^{2}-b / 2,
\end{gathered}
$$

where we defined the aspect ratio $\epsilon=L_{y} / L_{x}$ and the dimensionless temperature $D=T / T_{R}$.

In the absence of a time-dependent applied bias, i.e., $F(t)=0$, it has been shown by a coarsening of the description [15-17] that the Langevin equation (1) can be reduced to an effective 1D Fokker-Planck equation, reading in dimensionless form

$$
\frac{\partial P(x, t)}{\partial t}=\frac{\partial}{\partial x}\left\{D \frac{\partial P}{\partial x}+V^{\prime}(x, D) P\right\},
$$

where

$$
V(x, D)=-D \ln \left[\frac{2 D}{G} \sinh \left(\frac{G w(x)}{D}\right)\right],
$$

and the prime refers to the derivative with respect to $x$. This equation describes the motion of a Brownian particle in a bistable potential of entropic nature. Remarkably, the effective potential does not only depend on the energetic contribution of the force $G$, but also on the temperature and the geometry of the structure in a nontrivial way. Notably, for the vanishing width at the two opposite corners of the geometry in Fig. 1 this entropic potential approaches infinity, thus intrinsically accounting for a natural reflecting boundary. It is important to emphasize that this bistable potential was not present in the 2D Langevin dynamics, but arises due to the entropic restrictions associated to the confinement. In general, after the coarse graining the diffusion coefficient will depend on the coordinate $x$ as well, but since in our case $\left|w^{\prime}(x)\right| \ll 1$, this correction can be safely neglected, cf. Ref. [15,17-20].

It is interesting to analyze the two limiting situations that can be obtained upon varying the value of the ratio between the energy associated to the transversal force and the thermal energy. For the energy-dominated case, i.e., $G w(x) / D \gg 1$, Eq. (6) yields $V(x)=-G w(x)$ (neglecting irrelevant constants), thus recovering conventional energy controlled SR [1-3]. In the opposite limit, i.e., for $G w(x) / D \ll 1$, the corresponding entropic potential function reads $V(x, D)=-D \ln [2 w(x)]$.

Two-state approximation. - It is instructive to analyze the occurrence of stochastic resonance in the context of the two-state approximation. For a potential $V(x)$ with barrier height $\Delta V$ the escape rate of an overdamped Brownian particle from one well to the other in the presence of thermal noise, and in the absence of a force, is given by the overdamped Kramers rate [21-23], reading in dimensionless units,

$$
r_{K}(D)=\frac{\sqrt{V^{\prime \prime}\left(x_{\min }\right)\left|V^{\prime \prime}\left(x_{\max }\right)\right|}}{2 \pi} \exp \left(\frac{-\Delta V}{D}\right),
$$

where $V^{\prime \prime}$ is the second derivative of the effective potential function, and with $x_{\max }$ and $x_{\min }$ indicating the position of the maximum and minimum of the potential, respectively.

For the potential given by Eq. (6) and the shape defined by Eq. (2), the corresponding Kramers rate for transitions from one basin to the other reads, in dimensionless units,

$$
r_{K}(D)=\frac{G \epsilon}{\pi} \frac{\sqrt{2 \sinh (G b / D) \sinh [G(b+2 \epsilon) / D]}}{\sinh ^{2}[G(b+2 \epsilon) / 2 D]} .
$$

Spectral amplification.-The occurrence of stochastic resonance can be detected in the spectral amplification $\eta$ [22]. It is defined by the ratio of the power stored in the response of the system at frequency $\Omega$ and the power of the driving signal, and which for the periodically driven twostate model, cf. Ref. [1], is given in dimensionless units as

$$
\eta=\frac{1}{D^{2}} \frac{4 r_{K}^{2}(D)}{4 r_{K}^{2}(D)+\Omega^{2}} .
$$


Next we demonstrate the occurrence of the resonance in the spectral amplification which signals the phenomenon of Entropic Stochastic Resonance (ESR). We demonstrate that ESR is neither a peculiarity of the two-state approximation nor of the equilibration assumption used to derive the effective 1D kinetic equation.

In order to study the appearance of stochastic resonance we analyzed the response of the system to the applied sinusoidal signal in terms of the spectral amplification $\eta$. In the presence of an oscillating force $F(t)$ in the $x$ direction there is an additional contribution to the effective potential in Eq. (5) and the 1D kinetic equation in dimensionless units reads

$$
\frac{\partial P(x, t)}{\partial t}=\frac{\partial}{\partial x}\left\{D \frac{\partial P}{\partial x}+\left[V^{\prime}(x, D)-F(t)\right] P\right\} .
$$

The numerical integration of the 1D kinetic equation (10) was done by spatial discretization, using a Chebyshev collocation method, and employing the method of lines to reduce the kinetic equation to a system of ordinary differential equations, which was solved using a backward differentiation formula method. This results in the timedependent probability distribution $P(x, t)$ and the timedependent mean value, defined as

$$
\langle x(t)\rangle=\int x P(x, t) d x .
$$

In the long-time limit this mean value approaches the periodicity of driving [22] with angular frequency $\Omega$. After a Fourier expansion of $\langle x(t)\rangle$ one finds the amplitude $M_{1}$ of the first harmonic of the output signal. Hence, the spectral amplification $\eta$ for the fundamental oscillation reads:

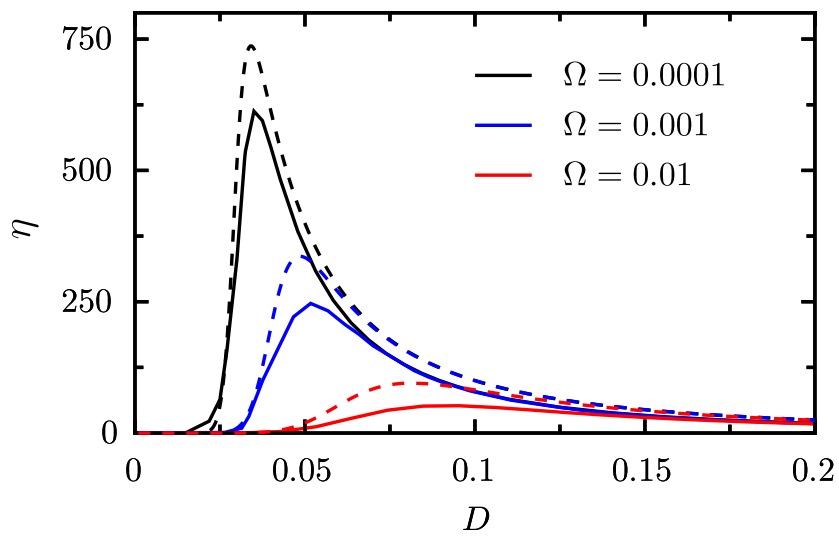

FIG. 2 (color online). In dimensionless units, the dependence of the spectral amplification $\eta$ on noise level $D$ for different driving frequencies, for the transversal force $G=1.0$, the driving amplitude $F_{0}=10^{-4}$, and for the width function $w(x)=$ $-\epsilon x^{4}+2 \epsilon x^{2}+0.01$ with the aspect ratio $\epsilon=1 / 4$. The solid lines correspond to the 1D modelling, cf. Eq. (10) and (12), whereas the dashed lines correspond to the two-state approximation, cf. Eq. (9).

$$
\eta=\left[\frac{M_{1}}{F_{0}}\right]^{2}
$$

The comparison of the 1D modeling and the two-state approximation in terms of the spectral amplification $\eta$, cf. Eqs. (9) and (12), demonstrates the capability of the twostate approximation for small driving frequencies and amplitudes, cf. Fig. 2.

$1 D$ modelling vs precise numerics $(2 D)$. - - In order to check the accuracy of the description we compared the results obtained by the $1 \mathrm{D}$ modelling with the results of Brownian dynamic simulations, performed by integration of the overdamped Langevin equation (1), for a 2D structure (see Fig. 1) whose shape is described by Eq. (2). In our case we have used the aspect ratio $\epsilon=1 / 4$ and the bottleneck width $b=0.02$. The simulations were carried out by the use of the standard stochastic Euler algorithm.

Figure 3 depicts the dependence of the spectral amplification $\eta$ on the noise strength for different values of the driving frequency, the driving amplitude and the value of $G$. It is important to point out that the results obtained from the 1D modelling (lines) are in excellent agreement with the numerical simulations of the full (2D) system (symbols) within a small relative error. This agreement is due to the fact that the considered potential function is a smooth function $\left(\left|w^{\prime}(x)\right| \ll 1\right)$, and in this situation our employed $1 \mathrm{D}$ approximation is expected to become very accurate $[15,17,18]$.

Figure 3(a) shows the dependence of the spectral amplification $\eta$ on the noise strength $D$ for various driving frequencies at a fixed transversal force and forcing amplitude $F_{0}$. Here, we observe an increase in the spectral amplification which signals the effect of stochastic resonance in the presence of entropic barriers. As observed for the usual "energy-dominated" SR [1] the resonance peak is more pronounced as the applied angular frequency $\Omega$ of the input signal decreases. Similarly, Fig. 3(b) depicts how ESR depends on the strength of the transversal force $G$. Interestingly, the maximal amplification increases upon decreasing the strength $G$ of the transversal force. However, the presence of the transversal force $G$ is crucial for observing a nonmonotonic behavior of the spectral amplification with increasing noise level $D$. In the limit of $G \rightarrow 0$, the spectral amplification increases monotonically with decreasing noise level and tends asymptotically to $1 / \Omega^{2}$ at small driving amplitudes $F_{0}$. Finally, Fig. 3(c) displays the dependence of the spectral amplification $\eta$ on the noise strength $D$ for various amplitudes of the driving force $F_{0}$ at a fixed value of the transversal force and driving frequency. Both the amplification of the signal and the optimal value of the noise at which it occurs increase as the driving amplitude decreases.

This novel ESR effect is characterized by the appearance of a maximum in the spectral amplification as a function of the noise strength $D$, just as in conventional energydominated SR [1]. However, in biological and practical 


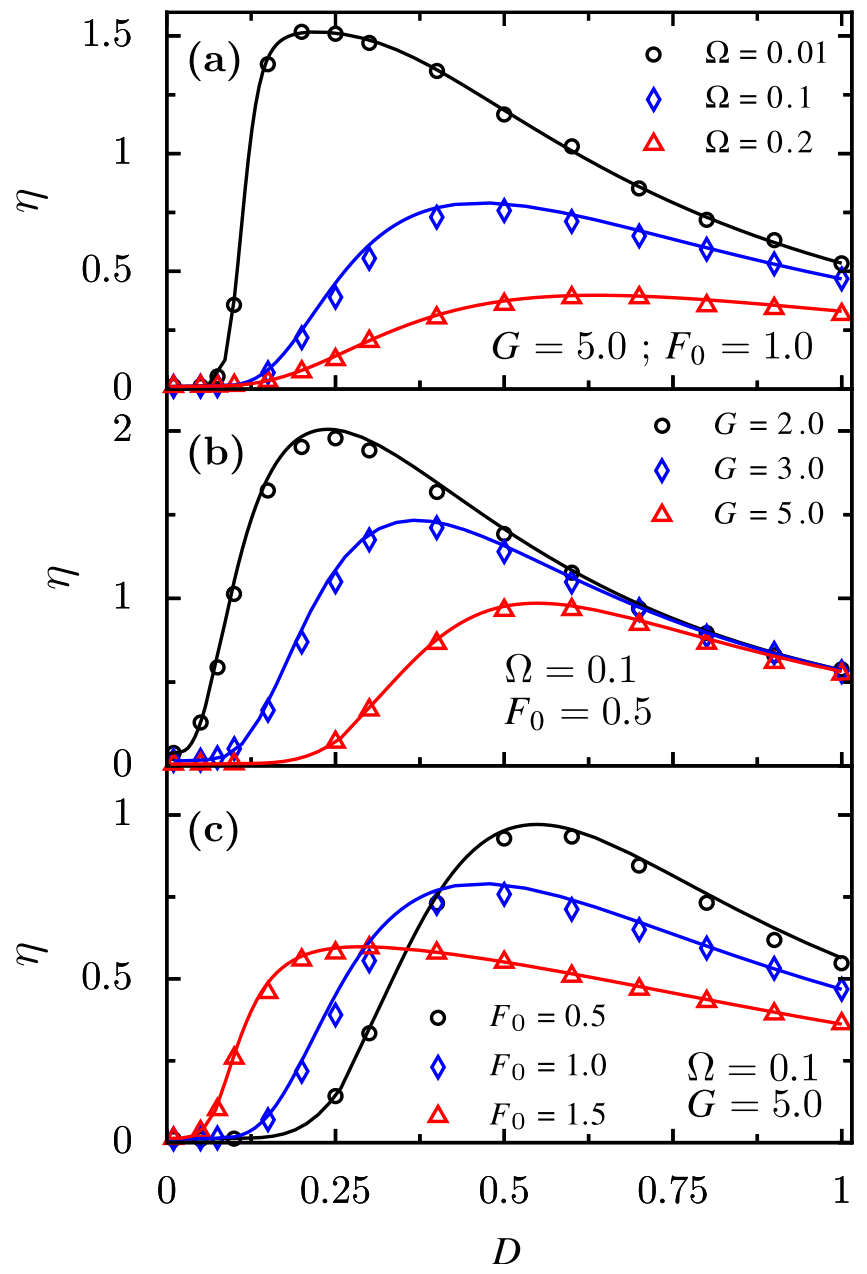

FIG. 3 (color online). In dimensionless units, the dependence of the spectral amplification $\eta$ on noise level $D$ for various values of the quantities, the driving amplitude $F_{0}$, driving frequency $\Omega$, and the transversal force $G$. The symbols correspond to the results of the Langevin simulations for the twodimensional structure with the shape defined by the dimensionless function $w(x)=-\epsilon x^{4}+2 \epsilon x^{2}+0.01$ with the aspect ratio $\epsilon=1 / 4$, whereas the lines are the results of the numerical integration of the 1D kinetic equation (10).

systems the temperature which controls the strength of the thermal noise is not a readily variable parameter. Our results suggest that, for a given temperature, a proper choice of the externally controlled parameters (i.e., the nature of the driving force, its amplitude and driving frequency, and the strength of the transversal force) might bring the system into an optimal regime where confinement and noise mutually interplay to boost noise-assisted transport inside a corrugated structure.

In conclusion, we have elucidated a new mechanism leading to the appearance of noise-induced resonant effects when a Brownian particle moves in a confined medium in the presence of periodic driving. The constrained motion impedes the access of the particle to certain regions of space and can be described in terms of a bistable entropic potential. The activated dynamics of the particle in this effective potential then results in a cooperative effect between noise and external modulation, yielding an entropic stochastic resonance. The effect detected is genuine for small-scale systems in which shape and fluctuations are unavoidable factors ruling their evolution. The advantageous possibilities of ESR on what concerns optimization and control may provide new perspectives in the understanding of systems at the scales of micrometers and nanometers and open new avenues in their manipulation and control.

This work has been supported by the DFG via research center, SFB-486, project A10, the Volkswagen Foundation (project I/80424), the German Excellence Initiative via the Nanosystems Initiative Munich (NIM), and by the DGCyT of the Spanish government through Grant No. FIS200501299.

[1] L. Gammaitoni et al., Rev. Mod. Phys. 70, 223 (1998).

[2] A. R. Bulsara and L. Gammaitoni, Phys. Today 49, No. 3, 39 (1996).

[3] P. Hänggi, Chem. Phys. Chem. 3, 285 (2002).

[4] J. M. G. Vilar and J. M. Rubí, Phys. Rev. Lett. 77, 2863 (1996); J. M. G. Vilar and J. M. Rubí, Phys. Rev. Lett. 78, 2886 (1997).

[5] V.S. Anishchenko et al., Phys. Usp. 42, 7 (1999).

[6] G. Schmid et al., Europhys. Lett. 56, 22 (2001).

[7] T. Wellens et al., Rep. Prog. Phys. 67, 45 (2004).

[8] H. Yasuda et al., Phys. Rev. Lett. 100, 118103 (2008).

[9] C. R. Nicolis and G. Nicolis, Scholarpedia 2, 1474 (2007).

[10] I. Goychuk and P. Hänggi, Phys. Rev. Lett. 91, 070601 (2003); I. Goychuk et al., Phys. Rev. E 71, 061906 (2005).

[11] B. Hille, Ion Channels of Excitable Membranes (Sinauer, Sunderland, 2001).

[12] R. M. Barrer, Zeolites and Clay Minerals as Sorbents and Molecular Sieves (Academic Press, London, 1978).

[13] L. Liu et al., Nature (London) 397, 141 (1999).

[14] A. M. Berezhkovskii and S. M. Bezrukov, Biophys. J. 88, L17 (2005).

[15] D. Reguera and J. M. Rubí, Phys. Rev. E 64, 061106 (2001).

[16] D. Reguera et al., Phys. Rev. Lett. 96, 130603 (2006).

[17] P. S. Burada et al., Phys. Rev. E 75, 051111 (2007).

[18] R. Zwanzig, J. Phys. Chem. 96, 3926 (1992).

[19] P. Kalinay and J. K. Percus, Phys. Rev. E 74, 041203 (2006).

[20] A. M. Berezhkovskii et al., J. Chem. Phys. 126, 134706 (2007).

[21] B. McNamara and K. Wiesenfeld, Phys. Rev. A 39, 4854 (1989).

[22] P. Jung and P. Hänggi, Phys. Rev. A 44, 8032 (1991); Europhys. Lett. 8, 505 (1989).

[23] P. Hänggi et al., Rev. Mod. Phys. 62, 251 (1990). 